\title{
Kleine-Levin syndrome: a review
}

\author{
This article was published in the following Dove Press journal: \\ Nature and Science of Sleep \\ 20 January 2014 \\ Number of times this article has been viewed
}

\author{
Mitchell G Miglis \\ Christian Guilleminault \\ Stanford University Sleep Medicine \\ Division, Stanford Outpatient Medical \\ Center, Redwood City, CA, USA
}

\begin{abstract}
Kleine-Levin syndrome is a recurrent hypersomnia associated with symptoms of hyperphagia, hypersexuality, and cognitive impairment. This article reviews the current available research and describes common clinical symptoms, differential diagnosis, and acceptable workup and treatment. Although deficits have traditionally been thought to resolve between episodes, functional imaging studies and long-term neuropsychological testing in select patients have recently challenged this notion. This may suggest that Kleine-Levin syndrome is not as benign as previously considered.
\end{abstract}

Keywords: Kleine-Levin syndrome, hypersomnia, adolescent sleep disorder, hypersexuality

\section{Introduction}

Kleine-Levin syndrome is a recurrent hypersomnia characterized by episodes of hypersomnia separated by intervening periods of normal behavior. In addition to hypersomnia, at least one of the following symptoms must be present: cognitive or mood disturbances, hyperphagia with compulsive eating, hypersexuality, or abnormal behavior such as irritability, aggression, or personality changes (Table 1).

Kleine-Levin syndrome was first described by Kleine in 1925 and elaborated on by Levin in 1936, but it was Critchley and Hoffman who further defined the syndrome in 1942 and gave it its eponym. ${ }^{1}$ In their classic paper "The Syndrome of Periodic Somnolence and Morbid Hunger"' they describe the cases of two men in their 20s who developed hypersomnia and hyperphagia, with symptoms lasting days to weeks at a time and recurring every few months. One of these patients developed his symptoms 6 months after a vaccination. There was no precipitating factor in the other. The authors postulated that a potential mechanism could involve a form of mild encephalitis affecting the hypothalamus and the frontal lobes.

In 1990, Kleine-Levin syndrome was classified by the International Classification of Sleep Disorders (ICSD). It was revised to its current state in 2005 with the release of the ICSD - revision 2 (ICSD-2). ${ }^{2}$

\section{Epidemiology}

Kleine-Levin syndrome is rare, affecting an estimated 1-5 per million individuals. ${ }^{3}$ There are only approximately 200 reported cases to date in the literature. It is a disease predominantly of teenagers, and boys are four times more likely to be affected than girls. It is rare for patients over the age of 30 years to present with their first episode, and, although cases have been reported, some authors question the existence 
Table I Diagnostic criteria for recurrent hypersomnia and Kleine-Levin syndrome (ICSD-2)

\section{Recurrent hypersomnia}

- Recurrent episodes of excessive daytime sleepiness lasting for 2 days to 4 weeks

- Episodes recur at least once per year

- Alertness, cognitive function and behavior are normal between episodes

- Hypersomnia is not explained by another sleep, medical, neurological, or psychiatric disorder, medication use, or substance abuse

\section{Kleine-Levin syndrome}

In addition to the recurrent hypersomnia criteria, the patient should also have at least one of the following

- Cognitive abnormalities - ex confusion, derealization, hallucinations

- Abnormal behavior - irritability, aggression

- Hyperphagia

- Hypersexuality

Abbreviation: ISCD-2, International Classification of Sleep Disorders - revison 2.

of an adult-onset form of the disease. In a collection of 239 patients, the median age of onset was 15 years in both males and females, with an age range of 4-80 years in males and 4-69 years in females. ${ }^{4}$ Prevalence is slightly higher in the Ashkenazi Jewish population. ${ }^{3}$

Overall, $5 \%$ of cases have been reported within family members, ${ }^{5}$ suggesting some degree of increased genetic susceptibility; however, no genetic abnormality has been yet identified. Two cases of monozygotic twins with Kleine-Levin syndrome have been described. ${ }^{6,7}$ In these cases, both sets of twins were positive for the DQB $1 * 0302 / * 0601$ allele.

In a large cohort of 108 patients diagnosed with Kleine-Levin syndrome, $25 \%$ had a complicated birth history and $15 \%$ had some degree of developmental delay. ${ }^{5}$ The significance of this is unclear, and, while some authors have theorized that such perinatal insults may predispose certain individuals to develop the disease later in life, there is no clear evidence to support this.

\section{Clinical presentation}

The symptoms of Kleine-Levin syndrome are characterized by their intermittent and periodic nature. During episodes, patients complain of excessive daytime sleepiness, despite sleeping anywhere from 12 to 21 hours per day. While awake, patients are often apathetic and report impairment in communication, concentration, and memory.

The classic triad of hypersomnia, hyperphagia, and hypersexuality is not always present. In fact, in one large case series, only $45 \%$ of patients presented with all three symptoms. ${ }^{8}$ More commonly, patients exhibit some form of cognitive impairment. Many patients report a feeling of derealization or "déjà vu" during episodes, as if they are in a dream-like state or experiencing life outside of their own bodies. In our experience this is the most common symptom next to hypersomnia. Altered perception, such as temperature sensation, has also been reported, as has pain insensitivity. ${ }^{5}$

Patients tend to eat compulsively and in large amounts. They favor sweets and will eat almost everything that is presented to them. Hypersexuality is seen more frequently in boys than in girls and takes the form of increased sexual drive, sexual comments, unwanted sexual advances, and frequent masturbation. Repetitive or compulsive behaviors are also common. Patients may become aggressive, especially if they are prevented from eating. Verbal outbursts may take the form of rude, offensive, and sexually charged language. Many patients are ashamed of their actions once the episode resolves and they revert to their normal behavior, and, as a result, may be hesitant to discuss their symptoms. Patients may also experience partial amnesia to the episodes.

Patients often withdraw from social interaction, and transient depression and anxiety can occur. As a result, patients are often misdiagnosed with psychiatric conditions, especially early in the disease course. Suicidal ideation and suicide attempts, while rare, have been reported. ${ }^{5}$ It is unclear if these cases represented true Kleine-Levin syndrome or were in fact cases of atypical depression. Nonetheless, the caregiver should be aware of this possibility and monitor the patient closely for any signs of suicidal behavior. In our experience, if this does occur, it seems to occur more frequently toward the end of an episode.

Patients may be difficult to wake in the early stages of the symptomatic period; however, toward the end of the episode they may appear to be simply resting or awake with their eyes closed. Some sleep studies have demonstrated that, if allowed to sleep at least 12 hours, patients with Kleine-Levin syndrome will spend a good portion of this time awake with their eyes closed in a state more akin to apathy than to sleep. ${ }^{9}$

Episodes progress fairly rapidly from time of onset and reach their peak symptomatic period within 24 hours. The episodes themselves usually last between 1 and 3 weeks, ${ }^{3}$ with a cycle length (time from onset of one episode to the onset of the next) of 60 to 100 days. ${ }^{4}$ Toward the end of an episode, the patient may complain of insomnia, and, once the symptoms have resolved completely, a state of profound relief and elation may occur, sometimes bordering on hypomania. 
Patients often report a triggering event prior to the onset of symptoms. Of all the reported triggers, infection and fever are by far the most common; ${ }^{10}$ alcohol intake, sleep deprivation, vaccination, head trauma, and dental anesthesia have also been reported. ${ }^{11-13}$

Kleine-Levin patients frequently exhibit difficulty with communication, executive functioning, and working memory. While deficits have been classically thought of as transient in nature, recent evidence indicates that some patients may exhibit long-term memory deficits ${ }^{14}$ and visuospatial dysfunction. ${ }^{12}$ Patients may also report migraine headaches, even if they have no prior history of migraines. Transient autonomic dysfunction has been reported in several cases. ${ }^{16}$

Reoccurrences vary in frequency. Episodes tend to recur more often toward the beginning of symptom onset, often several times in the course of a single year. As the disorder progresses and the patient ages, the cycle length increases; however, rarely does it extend beyond a year. Symptoms tend to wane in severity as the disorder progresses and eventually spontaneously resolve in patients with adolescent onset. In patients with adult onset, the disorder is less likely to resolve. In one large series, the median duration of symptoms was 13.6 years. ${ }^{17}$ Longer duration was associated with male sex, the presence of hypersexuality, and adult onset of symptoms (greater than 20 years of age). Tables 2 and 3 list some of the key clinical points discussed.

\section{Differential diagnosis}

The clinician must rule out any other contributing medical, neurological, sleep, or psychiatric disorder, as well as any contributing medication or substance use. A proper neurological examination should be performed in all patients, including fundoscopic inspection to evaluate for signs of increased intracranial pressure.

Atypical depression is by far the most common initial diagnosis in these patients. However, unlike true depression, mood changes in Kleine-Levin syndrome tend to manifest acutely at symptom onset and disappear abruptly once the episode has resolved. If patients do experience psychotic symptoms such as delusions or hallucinations, they are

Table 2 Common symptoms in patients with Kleine-Levin syndrome

- Hypersomnia

- Derealization

- Apathy

- Disinhibition and hypersexuality

- Compulsive hyperphagia

- Altered perception and temperature sensation
Table 3 Kleine-Levin syndrome: key clinical points

- Symptoms of depersonalization and sensory disturbances are more common than disinhibition

- Hypersexuality is much more common in men than women

- Peak symptomatic period occurs within 24 hours and lasts anywhere between I and 3 weeks

- Recurrences vary in frequency but typically occur every 2-3 months

- Common triggers include infection, fever, alcohol intake, sleep deprivation, vaccination, menses, and head trauma

- Episodes tend to wane in frequency and severity as the disease progresses

- Depression and anxiety are common

- Most patients are amnestic to the episodes

- Patients may report persistent memory deficit

short-lived and parallel the duration of the symptomatic period.

Other forms of primary hypersomnia should be considered. Vivid dreaming can occur in Klein-Levin syndrome, as can hypnagogic and hypnopompic hallucinations. Sleep paralysis, rapid eye movement (REM) behavior disorder, and cataplexy are not features of Kleine-Levin syndrome and, if present, should prompt the clinician to consider an alternative diagnosis of narcolepsy.

Sleep disordered breathing should be considered in any patient with excessive daytime sleepiness, even if initially episodic in nature, and the examination should include evaluation of the nasal airway and oropharynx to assess for any sites of potential airway obstruction. If present, a polysomnogram should be performed.

Menstruation-related hypersomnia is a rare form of recurrent hypersomnia that may also be considered in the differential. ${ }^{18}$ There is some thought that this condition may represent a variant of Kleine-Levin syndrome; however, several key distinctions must be noted. Most importantly, the symptoms of disinhibition and cognitive impairment seen in Kleine-Levin patients are less frequent in those with menstruation-related hypersomnia. In addition, while patients with menstruation-related hypersomnia may experience multiple episodes per year concurrent with their menstrual cycle, rarely do patients with Kleine-Levin syndrome experience more than two to three episodes a year.

The differential diagnosis also includes drug or alcohol use, temporal lobe epilepsy, Klüver-Bucy syndrome secondary to bilateral temporal lobe lesions, mass lesions, metabolic encephalopathies such as mitochondrial disease or urea cycle defects, Lyme disease, and acute intermittent porphyria.

Workup should include a urine or serum drug screen, basic metabolic panel, urine and serum assays to test for 
inborn errors of metabolism, folate and B12 levels, Lyme serology, and, if clinically indicated, urine porphobilinogen. Imaging studies such as magnetic resonance imaging (MRI) are performed to rule out structural or vascular lesions, and an electroencephalography (EEG) can be performed to help rule out complex partial seizures. Lumbar puncture may be considered to rule out meningitis, especially if the patient is febrile on presentation. With the exception of the EEG, which may demonstrate generalized or frontotemporal slowing if performed during an episode, the above workup is frequently normal, and patients are often given a psychiatric diagnosis until several episodes are noted and the patient is referred to a physician who is familiar with the diagnosis of KleineLevin syndrome. Table 4 lists some of the diagnoses that should be considered.

\section{Etiology}

The etiology of Kleine-Levin syndrome is unknown. Many patients report a prodromal flu-like illness or upper respiratory tract infection (URI). In a recent study in Taiwanese patients, a significant correlation was found between Kleine-Levin episodes and preceding URI symptoms. ${ }^{10}$ The authors in this study reported that $96.6 \%$ of first episodes of Kleine-Levin syndrome occurred after URI symptoms, fever, or encephalitis. Recurrent events were triggered by infection (65\%), fever (20\%), psychological events/stress (5\%), and sleep deprivation (5\%). There was no association with vaccinations. No seasonal variation was found.

As is the case in narcolepsy with cataplexy, an autoimmune mechanism has been suggested; however, the evidence is much less robust in Kleine-Levin syndrome. The DQB1-0201 haplotype was found to be twice as frequent in a controlled study of 30 European patients; ${ }^{19}$ however, this has not been replicated in larger patient populations. $^{8}$

Table 4 Differential diagnosis

- Medication or substance use
- Primary psychiatric illness (ie, atypical depression, bipolar disorder)
- Sleep disordered breathing
- Narcolepsy
- Menstruation-related hypersomnia
- Complex partial seizures
- Klüver-Bucy syndrome (bilateral temporal lobe lesions)
- Metabolic encephalopathies
- Herpes simplex virus encephalitis
- Lyme disease
- Intermittent porphyria

\section{Polysomnography and electroencephalography}

EEG demonstrates generalized slowing of drowsiness in up to $70 \%$ of cases during episodes. ${ }^{20}$ Polysomnography (PSG) is for the most part unremarkable. In one study, PSG performed toward the first half of the symptomatic period demonstrated a decrease in slow wave sleep, and PSG performed toward the second half of the symptomatic period demonstrated a decrease in REM sleep. ${ }^{21}$ The quantifiable decrease in slow wave sleep during the first half was greater than the decrease in REM during the second half. Overall sleep efficiency was poor. Multiple sleep latency tests were then performed on a handful of these patients. There was no significant difference in either mean sleep latency or the number of sleep onset REM periods between symptomatic and asymptomatic periods.

\section{Serum and cerebrospinal fluid analysis}

Serological markers of inflammation are normal during episodes. There were no abnormalities identified on serum and cerebrospinal fluid (CSF) analysis in a series of four Kleine-Levin patients. ${ }^{22}$ CSF hypocretin-1, which is low or deficient in patients with narcolepsy with cataplexy (less than $110 \mathrm{pg} / \mathrm{mL}$ ), is within the normal range in Kleine-Levin patients. However, levels were slightly lower during episodes than between episodes, although still within the range of normal. This was corroborated in a more recent sample of 42 patients. ${ }^{23}$ In this study, Kleine-Levin patients had lower hypocretin levels during episodes than matched controls. In addition, these levels increased during the recovery period between episodes, leading the authors to postulate that hypocretin may play some role in the pathophysiology of Kleine-Levin syndrome. At the moment, however, CSF hypocretin has no clinical value in the diagnosis of KleineLevin syndrome.

\section{Imaging}

Brain morphology is typically normal on MRI and computed tomography (CT), although cortical and cerebellar atrophy has been reported in one adult patient. ${ }^{24}$ Atrophy may be more common in adult patients as well as those with longstanding disease; however, this has not been systematically studied. Functional imaging, on the other hand, has been more recently utilized and is frequently abnormal in Kleine-Levin patients. Several studies utilizing single photon emission CT scanning during patients' symptomatic periods have demonstrated hypoperfusion in the thalamus, hypothalamus, temporal 
lobes, orbitofrontal and parasagittal frontal lobes, and, less commonly, the basal ganglia and occipital regions. ${ }^{24-27}$ Thalamic hypoperfusion has been the most consistent finding in these studies when patients are examined during their symptomatic period. Once patients revert to their normal behavior, the thalamic hypoperfusion seems to resolve, while residual hypoperfusion persists in other brain locations. ${ }^{23}$

Fludeoxyglucose-positron emission tomography scanning has demonstrated asymmetric hypometabolism in the thalamus and hypothalamus. ${ }^{28}$ Researchers have also demonstrated reduced dopamine binding potential in the striatum during the symptomatic period. ${ }^{29}$

In patients with recurrent episodes, there is evidence that these imaging abnormalities may persist even after other symptoms resolve. Several studies have demonstrated persistent hypoperfusion in the mesial temporal lobe, frontal lobe, and basal ganglia of Kleine-Levin patients during asymptomatic periods. ${ }^{30,31}$ These deficits are more common in patients with longstanding disease.

It is conceivable that the temporal lobes abnormalities may correlate with memory deficits, the thalamic abnormalities with hypersomnia and circadian rhythm dysfunction, the orbitofrontal and anterior parasagittal areas abnormalities with the behavioral symptoms observed (apathy, disinhibition, aggression, hyperphagia, hypersexuality), and the mesial temporal abnormalities with the prominent sensation of derealization; however, these correlations have not been systematically evaluated.

Functional MRI studies and magnetic resonance spectroscopy studies have demonstrated a significant negative correlation between thalamic functional MRI-activity and thalamic $N$-acetylaspartic acid-concentration in KleineLevin patients while performing tasks involving working memory, ${ }^{32}$ suggesting increased effort in the Kleine-Levin syndrome patients upon performing the task. Working memory is important in the temporary storage and manipulation of information and is fundamental to many higher order processes such as comprehension, communication, visuospatial processing, and problem solving. All of these functions are significantly impaired in Kleine-Levin patients during episodes. ${ }^{33}$ It has been suggested that the thalamus is intricately involved in regulating working memory circuits. $^{34}$

All of these studies add to the growing body of literature that supports the concept of a disorder affecting multiple areas of the brain in both cortical and subcortical regions. The earlier notion that Kleine-Levin syndrome is simply a disorder of the hypothalamus is no longer supportable in light of this current evidence. In addition, several studies have demonstrated that deficits can persist long after the symptomatic period has ended, leading to long-term neuropsychological impairment.

\section{Neuropsychological testing}

The deficits in working memory that patients frequently exhibit during episodes can manifest as impairment in immediate and delayed verbal and visual recall. ${ }^{15}$ These deficits have been quantified in studies utilizing the Wechsler Memory Scale, on which Kleine-Levin patients typically perform poorly. ${ }^{35}$ These memory impairments may persist long after resolution of an episode, even after perceived resolution of the disorder itself. In one patient with asymmetric frontotemporal hypoperfusion as demonstrated on single photon emission computed tomography scanning performed during an episode, short-term verbal and visual memory deficits were present 6 years after his final symptomatic period. ${ }^{35}$ In this particular series of patients, the duration of illness ranged from 2.5 to 9 years. Table 5 reviews ancillary testing in Kleine-Levin syndrome.

\section{Autopsy}

There are few reported autopsy cases in the literature. Inflammatory infiltrates were observed in the hypothalamus in two patients ${ }^{36,37}$ and in the thalamus in two patients. ${ }^{36,38}$ One patient demonstrated only mild hypopigmentation of the substantia nigra. ${ }^{39}$

Table 5 Ancillary testing in Kleine-Levin syndrome

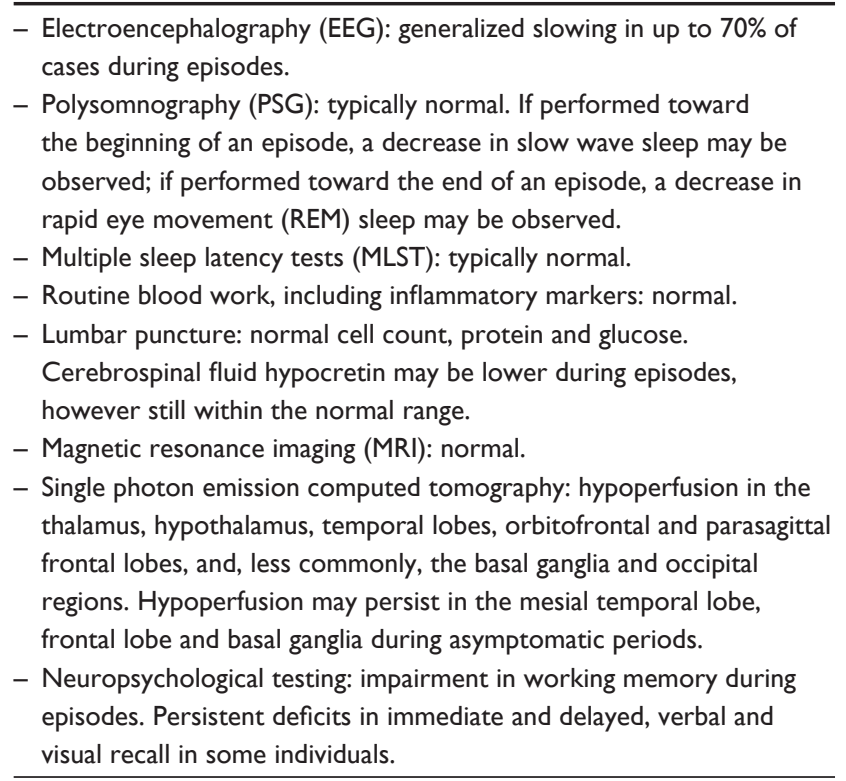




\section{Treatment}

No medication has been convincingly demonstrated to be efficacious in the treatment of Kleine-Levin syndrome. Unless the episodes are particularly severe, the best treatment is supportive. Educating parents and refraining from aggressive treatment is often the recommendation of choice. The patient should be allowed to rest at home in a safe and comfortable environment. School and work activities should be postponed or adjusted until the symptoms have resolved. The patient should not be allowed to wander unattended, drive a car, or operate heavy machinery. Symptoms of depression or anxiety should be monitored closely and treated if present.

Stimulants may be beneficial for symptomatic treatment if utilized early in the symptomatic cycle. Modafinil (Teva Pharmaceutical Indsutries Ltd., Fraser, PA, USA), has been reported to ameliorate symptoms in some reports. ${ }^{19,40,41}$ It should be noted that, while stimulants may reduce the duration of the symptomatic period, they do not reduce recurrence rate. In addition, there are some reports of stimulant-induced paradoxical agitation. ${ }^{42}$ If this does occur, it is more likely to occur if medication is taken toward the end of an episode, when patients are less apathetic and more easily agitated. Antipsychotics such as risperidone have been used for prolonged psychotic symptoms. ${ }^{8,17}$ Benzodiazepines may be used for treatment of anxiety. In one series, amantadine had reported effect as a prophylactic agent, discontinuing episodes in $42 \%$ of patients when administered on the first day of symptoms. ${ }^{8}$

Lithium has also been proposed as a preventative agent; ${ }^{43}$ however, quality evidence is lacking. It is often difficult to exclude depression in the patient populations examined and the results of these studies should be interpreted with caution. It is worth noting that only lithium had a reported response rate significantly higher than medical abstention (odds ratio $=3.8, P=0.02$ ). ${ }^{17}$ On the other hand, the Taiwan group has tried lithium on a long-term basis in their adolescent population and were unable to obtain control or reduce recurrence rate at 2-year follow-up (YS Huang, personal communication, August 2013).

Other mood stabilizers and antiepileptics studied have included valproic acid ${ }^{44}$ carbamazepine, ${ }^{45}$ phenytoin, gabapentin, ${ }^{46}$ and lamotrigine. None of these have consistently demonstrated significant benefit. Antidepressants including tricyclics, selective serotonin reuptake inhibitors, and serotonin-norepinephrine reuptake inhibitors have not been shown to have any prophylactic effect either. One patient with refractory symptoms has been reported to show a favorable response to sodium oxybate. ${ }^{47}$ It should be stated that none of the drug trials have been double-blinded or placebo controlled. In fact, this led the authors of a recent Cochrane Review to withhold recommendation of any of the medications utilized in the treatment of Kleine-Levin syndrome. ${ }^{48}$ Out of 257 studies initially identified, 31 had the potential to be included. Ultimately, none of these studies met the eligibility criteria for inclusion. Table 6 lists some of the treatment options discussed.

\section{Summary and future directions}

Kleine-Levin syndrome is a fascinating periodic hypersomnia whose underlying etiology remains to be elucidated. Key symptoms include hypersomnia and cognitive impairment. The classic triad of hypersomnia, hyperphagia, and hypersexuality is not always present. Derealization is a prominent component.

Although an autoimmune mechanism has been suggested, there are likely heterogeneous factors at play in certain susceptible individuals. When combined with a precipitating event such as a minor infection, a transient multifocal encephalopathy ensues. It has been proposed that such a precipitating event may lead to transient permeability of the blood-brain barrier, thus predisposing these individuals to recurrent events. An animal model would add much to the understanding of the underlying pathophysiology. This is yet to be developed and should be considered in future research.

While several familial cases have been identified, genetic studies have been thus far unrevealing. The association with a specific human leukocyte antigen genotype has not been

Table 6 Treatment of Kleine-Levin syndrome

\section{A. Supportive}

I. Allow patient to rest at home under supervision in a safe and comfortable environment.

2. Postpone or adjust school and work activities to accommodate long sleep times.

3. Do not allow the patient to wander unattended or to operate a car or heavy machinery.

4. Monitor for symptoms of depression or anxiety.

5. Between episodes, maintain consistent sleep-wake schedules, avoid alcohol and sick contacts.

B. Medication

I. Consider amantadine if patient presents in the early stages of an episode.

2. Consider modafinil for excessive daytime sleepiness in the early symptomatic period.

3. Consider short-term course of an antidepressant, mood stabilizer, anxiolytic or antipsychotic for psychiatric symptoms.

Note: Modafinil, Teva Pharmaceuticals Indsutries Ltd., Frazer, PA, USA. 
consistently demonstrated. Genomic mapping studies and linkage analyses should be considered using known familial cases in attempts to investigate a possible genetic basis for this disease.

Kleine-Levin syndrome, while rare, is often misdiagnosed or unrecognized, and should be considered in any teenager presenting with recurrent episodes of hypersomnia concurrent with cognitive changes, depersonalization, or disinhibition. Atypical depression and substance use must be ruled out before a diagnosis of Kleine-Levin is considered. Treatment is generally supportive, though certain stimulant medications such as modafinil may help to alleviate symptoms if utilized early in the symptomatic period. Although deficits have traditionally been thought to resolve between episodes, functional imaging studies and long-term neuropsychological testing in select patients have more recently challenged this notion. This suggests that Kleine-Levin syndrome is not as benign as previously considered. More long-term follow-up studies are needed, with special emphasis on residual neuropsychological impairment and the risk factors involved in these patients.

\section{Disclosure}

The authors report no conflicts of interest in this work.

\section{References}

1. Critchley M, Hoffman H. The syndrome of periodic somnolence and morbid hunger (Kleine-Levin syndrome). Br Med J. 1942;1: 137-139.

2. American Academy of Sleep Medicine. International Classification of Sleep Disorders: Diagnostic and Coding Manual. 2nd ed. Westchester, IL: American Academy of Sleep Medicine; 2005.

3. Frenette E, Kushida CA. Primary hypersomnias of central origin. Semin Neurol. 2009;29(4):354-367.

4. Billiard M, Jaussent I, Dauvilliers Y, Besset A. Recurrent hypersomnias: a review of 339 cases. Sleep Med Rev. 2011;15:247-257.

5. Arnulf I, Rico TJ, Mignot E. Diagnosis, disease course, and management of patients with Kleine-Levin syndrome. Lancet Neurol. 2012;11(10): 918-928.

6. Ueno T, Fukuhara A, Ikegami A, Ohishi F, Kume K. Monozygotic twins concordant for Kleine-Levin syndrome. BMC Neurol. 2012;12:31.

7. Peraita-Adrados R, Vicario JL, Tafti M, García de León M, Billiard M. Monozygotic twins affected with Kleine-Levin syndrome. Sleep. 2012;35(5):595-596.

8. Arnulf I, Lin L, Gadoth N, et al. Kleine-Levin syndrome: a systematic study of 108 patients. Ann Neurol. 2008;63:482-493.

9. Ugoljew A, Kurella B, Nickel B. [Sleep polygraphic studies as an objective method for assessing the therapeutic result in a case of periodic hypersomnia (Kleine-Levin syndrome)]. Nervenarzt. 1991;62(5): 292-297. German.

10. Huang YS, Guilleminault C, Lin KL, Hwang FM, Liu FY, Kung YP. Relationship between Kleine-Levin syndrome and upper respiratory infection in Taiwan. Sleep. 2012;35(1):123-129.

11. Billiard M, Podesta C. Recurrent hypersomnia following traumatic brain injury. Sleep Med. 2013;14(5):462-465.

12. Pike M, Stores G. Klein-Levin syndrome: A cause of diagnostic confusion. Arch Dis Child. 1994;71:355-357.
13. Ferguson BG. Kleine-Levin syndrome: a case report. J Child Psychol Psychiatry. 1986;27(2):275-278.

14. Landtblom AM, Dige N, Schwerdt K, Säfström P, Granérus G. Short-term memory dysfunction in Kleine-Levin syndrome. Acta Neurol Scand. 2003;108:363-367.

15. Körtner K, Hansen ML, Danker-Hopfe H, Neuhaus AH, JockersScherübl MC. Persistent deficits of visual recall in Kleine-Levin syndrome. J Clin Neurosci. 2011;18:439-440.

16. Hegarty A, Merriam AE. Autonomic events in Kleine-Levin syndrome. Am J Psychiatry. 1990;147:951-952.

17. Arnulf I, Zeitzer JM, File J, Farber N, Mignot E. Kleine-Levin syndrome: a systematic review of 186 cases in the literature. Brain. 2005;128:2763-2776.

18. Billard M, Guilleminault C, Dement WC. A menstruation-linked periodic hypersomnia: Kleine-Levin syndrome or new clinical entity. Neurology. 1975;25:436-443.

19. Dauvilliers Y, Mayer G, Lecendreux M, et al. Kleine-Levin syndrome: an autoimmune hypothesis based on clinical and genetic analyses. Neurology. 2002;59:1739-1745.

20. Papacostas SS, Hadjivasilis V. The Kleine-Levin syndrome. Report of a case and review of the literature. Eur Psychiatry. 2000;15(4): 231-235.

21. Huang YS, Lin YH, Guilleminault C. Polysomnography in Kleine-Levin syndrome. Neurology. 2008;70:795-801.

22. Dauvilliers Y, Baumann CR, Carlander B, et al. CSF hypocretin-1 levels in narcolepsy, Kleine-Levin syndrome, and other hypersomnias and neurological conditions. J Neurol Neurosurg Psychiatry. 2003;74(12): 1667-1673.

23. Qinghua L. CSF hypocretin levels in patients with Kleine-Levin syndrome: Proceedings of the 5th World Congress on Sleep Medicine, Valencia, Spain, September 28-October 2, 2013. In Press 2013.

24. Shi YT, Tang BS, Jiang H. Kleine-Levin syndrome with brain atrophy. J Clin Neurosci. 2013;20(7):1027-1028.

25. Huang YS, Guilleminault C, Kao PF, Liu FY. SPECT findings in the Kleine-Levin syndrome. Sleep. 2005;28(8):955-960.

26. Haba-Rubio J, Prior JO, Guedj E, Tafti M, Heinzer R, Rossetti AO. Kleine-Levin syndrome: functional imaging correlates of hypersomnia and behavioral symptoms. Neurology. 2012;79(18):1927-1929.

27. Hong SB, Joo EY, Tae WS, Lee J, Han SJ, Lee HW. Episodic diencephalic hypoperfusion in Kleine-Levin syndrome. Sleep. 2006;29(8): 1091-1093.

28. Lo YC, Chou YH, Yu HY. PET finding in Kleine-Levin syndrome. Sleep Med. 2012;13(6):771-772.

29. Hoexter MQ, Shih MC, Felício AC, Tufik S, Bressan RA. Greater reduction of striatal dopamine transporter availability during the symptomatic than asymptomatic phase of Kleine-Levin syndrome. Sleep Med. 2010;11(9):959.

30. Landtblom AM, Dige N, Schwerdt K, Säfström P, Granérus G. A case of Kleine-Levin syndrome examined with SPECT and neuropsychological testing. Acta Neurol Scand. 2002;105(4):318-321.

31. Landtblom AM, Dige N, Schwerdt K, Säfström P, Granérus G. Short-term memory dysfunction in Kleine-Levin syndrome. Acta Neurol Scand. 2003;108(5):363-367.

32. Vigren P, Tisell A, Engström M, et al. Low Thalamic NAA-Concentration Corresponds to Strong Neural Activation in Working Memory in KleineLevin Syndrome. PLoS One. 2013;8(2):e56279.

33. Engström M, Landtblom AM, Karlsson T. Brain and effort: brain activation and effort-related working memory in healthy participants and patients with working memory deficits. Front Hum Neurosci. 2013;7: 140.

34. Lim J, Choo WC, Chee MW. Reproducibility of changes in behaviour and fMRI activation associated with sleep deprivation in a working memory task. Sleep. 2007;30(1):61-70.

35. Sagar RS, Khandelwal SK, Gupta S. Interepisodic morbidity in Kleine-Levin syndrome. Br J Psychiatry. 1990;157:139-141.

36. Fenzi F, Simonati A, Crosato F, Ghersini L, Rizzuto N. Clinical features of Kleine-Levin syndrome with localized encephalitis. Neuropediatrics. 1993;24(5):292-295. 
37. Takrani LB, Cronin D. Kleine-Levin syndrome in a female patient. Can Psychiatr Assoc J. 1976;21(5):315-318.

38. Carpenter S, Yassa R, Ochs R. A pathologic basis for Kleine-Levin syndrome. Arch Neurol. 1982;39(1):25-28.

39. Koerber RK, Torkelson R, Haven G, Donaldson J, Cohen SM, Case M. Increased cerebrospinal fluid 5-hydroxytryptamine and 5-hydroxyindoleacetic acid in Kleine-Levin syndrome. Neurology. 1984;34(12): $1597-1600$.

40. Aggarwal A, Garg A, Jiloha RC. Kleine-Levine syndrome in an adolescent female and response to modafinil. Ann Indian Acad Neurol. 2011;14(1):50-52.

41. Huang YS, Lakkis C, Guilleminault C. Kleine-Levin syndrome: current status. Med Clin North Am. 2010;94(3):557-562.

42. Mignot, EJ. A Practical Guide to the Therapy of Narcolepsy and Hypersomnia Syndromes. Neurotherapeutics. 2012;9:739-752.
43. Muratori F, Bertini N, Masi G. Efficacy of lithium treatment in Kleine-Levin syndrome. Eur Psychiatry. 2002;17(4):232-233.

44. Crumley FE. Valproic acid for Kleine-Levin syndrome. J Am Acad Child Adolesc Psychiatry. 1997;36(7):868-869.

45. Mukaddes NM, Kora ME, Bilge S. Carbamazepine for Kleine-Levin syndrome. J Am Acad Child Adolesc Psychiatry. 1999;38(7): 791-792.

46. Itokawa K, Fukui M, Ninomiya M, et al. Gabapentin for Kleine-Levin Syndrome. Intern Med. 2009;48(13):1183-1185.

47. Ortega-Albás JJ, Díaz JR, López-Bernabé R, Vera JF, Alós M, Serrano AL. Treatment of Kleine-Levin syndrome with sodium oxybate. Sleep Med. 2011;12(7):730.

48. Oliveira MM, Conti C, Saconato H, Fernandes do Prado G. Pharmacological treatment for Kleine-Levin syndrome. Cochrane Database Syst Rev. 2009;(2):CD006685.

\section{Publish your work in this journal}

Nature and Science of Sleep is an international, peer-reviewed, open access journal covering all aspects of sleep science and sleep medicine, including the neurophysiology and functions of sleep, the genetics of sleep, sleep and society, biological rhythms, dreaming, sleep disorders and therapy, and strategies to optimize healthy sleep. The journal welcomes

\section{Dovepress}

original research, clinical \& epidemiological studies, reviews \& evaluations, case reports and extended reports. The manuscript management system is completely online and includes a very quick and fair peerreview system, which is all easy to use. Visit http://www.dovepress.com/ testimonials.php to read real quotes from published authors. 